This document is published in:

Aleven, V. et al. (eds.) (2010). Intelligent Tutoring Systems: 10th International Conference, ITS 2010, Pittsburgh, PA, USA, June 14-18, 2010, Proceedings, Part II. (Lecture Notes in Computer Science, 6095). Springer, 384-386. DOI: http://dx.doi.org/10.1007/978-3-642-13437-1_78

(C) 2010 Springer-Verlag Berlin Heidelberg 


\title{
Behavior Effect of Hint Selection Penalties and Availability in an Intelligent Tutoring System
}

\author{
Pedro J. Muñoz-Merino, Carlos Delgado Kloos, and Mario Muñoz-Organero \\ Carlos III University of Madrid, Department of Telematics Engineering, Avda de la \\ Universidad, 30, E-28911 Leganés (Madrid), Spain \\ \{pedmume, munozm, cdk\} ait.uc3m.es
}

\begin{abstract}
This paper presents empirical results about the behavior effect of two different hinting strategies applied on exercises within an ITS: having some penalty on the scoring for viewing hints or not having any effect on the scoring; and hints directly available or only available as a result to an incorrect attempt. We analyze the students' behavior differences when these hinting techniques changed, taking into account the type and difficulty of the presented exercises.
\end{abstract}

Keywords: assessment, hints, empirical evaluation, student behavior.

\section{Introduction}

Different student behaviours within hinting systems have been studied. Paper [1] shows a model identifying the ideal student behavior. The article [2] shows different useful parameters based on student actions. The different student behaviors have an effect on their learning gains [1], and the different student actions have a relationship with their final scores on the tests [2].

This paper focuses on the analysis of the student behavior regarding the variation of two hinting strategies, taking into account the type of problem and their difficulty. These hinting techniques imply some effort or cost for obtaining help. Whether the provision of help to students is beneficial or not, is not a trivial question [3].

Some existing hinting systems (e.g. SIETTE [4]) can adapt hint contents. The results of this paper can be used for example for the adaptation of the commented hinting techniques without changing the hint contents.

\section{Preparation of the Experiment}

The experiment took place in two editions of a computer architecture laboratory course. The data was taken from the hinting module [5] of XTutor during two sessions. Students interacted with a set of exercises with hints, changing the hinting techniques to compare. Exercises were multiple Choice (MC, only one option is correct), Multiple Response (MR, the correct solution involves the selection of several options) and Fill In the Blank (FIB). Four exercises (S1, M1, I1, F1) changed the strategy of having penalties for viewing hints or not. Another four exercises (S2, M2, 
I2, F2) changed the strategy of having penalties for viewing hints, or not but with a maximum limit of hints to select. Finally, four exercises (S4, M4, I4, F4) changed the strategy of hint availability. Each student interacted only with one of these hinting techniques for a specific problem, and this is selected randomly at the beginning but in a way that there is a balance in the number of total presented hinting techniques.

\section{Results and Analysis}

Tables 1 and 2 show the statistics for the 12 exercises, comparing the hinting techniques regarding penalties for selecting hints (table 1) and hint availability (table 2). The tables distinguish the type of problem (MC, MR or FIB), their difficulty (in a scale from 0 to 4 ), the total number of interactions with each one of the techniques compared, the number of times a user selected at least one hint for each one of the techniques compared, and the probability of requesting at least one hint $(\mathrm{P})$ provided in a confidence interval for each one of the hinting techniques (applying the binomial non-parametric test with a $95 \%$ probability). If there is not an intersection of the intervals of the two techniques compared for a specific exercise, then there is a statistically significant difference between both techniques for this specific exercise. For each specific problem, samples are independent. We are planning to analyze more hinting techniques and integrate the results with a logistic regression model taking into account the random effect of the same student answering different exercises.

Table 1. Statistics for penalties or not for selecting hints, for the eight exercises

\begin{tabular}{lcccccccc}
\hline Problem & Type & Diff. & $\begin{array}{c}\text { Total } \\
\text { Interact. } \\
\text { with } \\
\text { Penalties }\end{array}$ & $\begin{array}{c}\text { Total } \\
\text { Interact. } \\
\text { without } \\
\text { Penalties }\end{array}$ & $\begin{array}{c}\text { Selected } \\
\text { Hints } \\
\text { with } \\
\text { Penalties }\end{array}$ & $\begin{array}{c}\text { Selected } \\
\text { Hints } \\
\text { without } \\
\text { Penalties }\end{array}$ & $\begin{array}{c}\text { P (\%) } \\
\text { Hint with } \\
\text { Penalties }\end{array}$ & $\begin{array}{c}\text { P (\%) } \\
\text { Hint } \\
\text { without } \\
\text { Penalties }\end{array}$ \\
\hline S1 & MC & 1 & 48 & 46 & 1 & 3 & {$[0,10]$} & {$[1,16]$} \\
M1 & FIB & 1 & 43 & 40 & 27 & 37 & {$[49,75]$} & {$[82,98]$} \\
I1 & FIB & 3 & 43 & 43 & 35 & 35 & {$[69,91]$} & {$[69,91]$} \\
F1 & MC & 3 & 42 & 47 & 7 & 13 & {$[8,29]$} & {$[17,40]$} \\
S2 & MR & 4 & 47 & 45 & 30 & 42 & {$[51,75]$} & {$[84,98]$} \\
M2 & MR & 3 & 42 & 42 & 25 & 34 & {$[45,73]$} & {$[68,90]$} \\
I2 & MR & 2 & 44 & 41 & 19 & 32 & {$[31,57]$} & {$[65,88]$} \\
F2 & MR & 1 & 37 & 36 & 11 & 11 & {$[18,45]$} & {$[18,45]$}
\end{tabular}

Table 2. Statistics for hints directly available or not for the four exercises

\begin{tabular}{lcccccccc}
\hline Problem & Type & Diff. & $\begin{array}{c}\text { Total } \\
\text { Interact. } \\
\text { no } \\
\text { available }\end{array}$ & $\begin{array}{c}\text { Total } \\
\text { Interact. } \\
\text { directly } \\
\text { available }\end{array}$ & $\begin{array}{c}\text { Selected } \\
\text { Hints } \\
\text { no } \\
\text { available }\end{array}$ & $\begin{array}{c}\text { Selected } \\
\text { Hints } \\
\text { directly } \\
\text { available }\end{array}$ & $\begin{array}{c}\text { P (\%) } \\
\text { Hint } \\
\text { no } \\
\text { available }\end{array}$ & $\begin{array}{c}\text { P (\%) } \\
\text { directly } \\
\text { available }\end{array}$ \\
\hline S4 & FIB & 3 & 39 & 46 & 23 & 34 & {$[45,72]$} & {$[61,84]$} \\
M4 & FIB & 4 & 36 & 40 & 27 & 37 & {$[60,86]$} & {$[82,98]$} \\
I4 & FIB & 3 & 28 & 41 & 19 & 29 & {$[51,82]$} & {$[57,82]$} \\
F4 & FIB & 1 & 30 & 37 & 6 & 22 & {$[9,36]$} & {$[44,73]$}
\end{tabular}


From table 1, there is a significant difference between the number of hints selected, comparing the cases of having penalties or not, when the amount of selected hints is not too high nor too low. As MC problems make the number of requested hints to be low then none of the cases resulted in a significant difference. As FIB problems make the number of requested hints to be high, then if the problem is difficult the amount of requested hints will be high, and there will not be significant difference; if it is easy or medium, then the number of requested hints will be medium and there will be significant difference. Finally, the MR problems have also certain effect that makes students to do a lot of attempts until they reach the correct answer, but they need much more attempts than for MC problems. So if the problem is easy then there will be few requested hints and the difference will not be significant, but if the problem is medium or difficult then the amount of requested hints will not be too high nor too low, so this can make the difference significant. A similar effect happens with the hinting technique of having hints directly available or not. In this case, it was only tested with FIB problems. A similar analysis can be performed from table 2 .

\section{Conclusions}

There were several statistically significant differences in the number of hint requests between the hinting technique of having penalties for selecting hints or not, depending on the type and difficulty of the problem. In a similar way, there was a statistically significant difference in the number of hint requests comparing hints directly available or not, depending on the problem difficulty. The probabilities for selecting hints were provided for each case, as confidence intervals.

Acknowledgments. Work partially funded by the Learn3 project TIN2008-05163/TSI within the Spanish "Plan Nacional de I+D+I", and the Madrid regional community project eMadrid S2009/TIC-1650.

\section{References}

1. Aleven, V., Mclaren, B., Roll, I., Koedinger, K.R.: Toward Meta-cognitive Tutoring: A Model of Help Seeking with a Cognitive Tutor. Int. J. Artif. Intell. 16, 101-128 (2006)

2. Feng, M., Heffernan, N.T., Koedinger, K.R.: Predicting state test scores better with intelligent tutoring systems: developing metrics to measure assistance required. In: Ikeda, M., Ashley, K.D., Chan, T.-W. (eds.) ITS 2006. LNCS, vol. 4053, pp. 31-40. Springer, Heidelberg (2006)

3. Beck, J.E., Chang, K., Mostow, J., Corbett, A.: Does Help Help? Introducing the Bayesian Evaluation. In: Woolf, B.P., Aïmeur, E., Nkambou, R., Lajoie, S. (eds.) ITS 2008. LNCS, vol. 5091, pp. 540-550. Springer, Heidelberg (2008)

4. Conejo, R., Guzmán, E., Pérez de-la Cruz, J.L., Millán, E.: An Empirical Study About Calibration of Adaptive Hints in Web-Based Adaptive Testing Environments. In: Wade, V.P., Ashman, H., Smyth, B. (eds.) AH 2006. LNCS, vol. 4018, pp. 71-80. Springer, Heidelberg (2006)

5. Muñoz-Merino, P.J., Delgado Kloos, C.: A software player for providing hints in problembased learning according to a new specification. Computer Applications in Engineering Education 17, 272-284 (2009) 